\title{
The world is graying: Dementia is an alarming issue
}

\author{
N Sapkota, Fellowship (Geriatric Psychiatry) \\ Associate Professor and Head, Department of Psychiatry \\ B.P. Koirala Institute of Health Sciences, Dharan, Nepal
}

The world's population is ageing hence the world is graying. The elderly population is substantially increasing not only in the high income countries but also in the low and middle income countries (LMICs) like Nepal in the past decade. As the world is graying, the chronic medical and neurological disease like dementia is expected to increase with elderly population growth. According to the WHO report ${ }^{1}$ between $2 \%$ and $10 \%$ of all cases of dementia start before the age of 65 . The prevalence doubles with every five-year increment in age after 65 . In other words, the prevalence of dementia is $5 \%$ for aged $65-70$, $10 \%$ for $75-80$, and even more after age 80 . As per this report the current estimates indicate 35.6 million people worldwide are living with dementia. This number is expected to double by 2030 and triple by 2050 . Dementia doesn't just affect individuals; it changes and affects the life of family members or the care takers. It has major burden for the family and leads financial implication. It is a costly condition in its social, economic and health dimensions. Nearly $60 \%$ of the burden of dementia is concentrated in low and middle income countries and this is likely to increase in coming years as the world is graying and ageing population is on the rise. The WHO report ${ }^{2}$ on dementia published in 2014 highlights the serious consequences of dementia in low and middle income countries and offers important statistics and key message for the policy makers ${ }^{2}$.

Elderly population in Nepal (age 60 years and above) is growing more than the general population. In 2001, there were 1.5 million older people in Nepal; this population has increased in 2011 to 2.7 million, accounting for 9\% of Nepal's population. The 2011 census has revealed that the rate of general population growth is $2.1 \%$ and $3.4 \%$ in the elderly. If the growth continues to increase at this rate, the population of senior citizens would become $3,779,772$ by 2021 . In the next ten years, over 1.3 million elderly populations will be added ${ }^{3}$. This would not only have great social implications but also on the health sector. This shift in the elderly cohort directly reflects to the increment in the number of dementia cases in the community as old age is an important risk factor for Dementia. In Nepal except hospital based study we don't have prevalence data but based on elderly population and at least 5 
percent prevalence of Dementia among 2.7 million elderly population in the country as per 2011 National census it can be estimated that there can be at least 135,000 suffering from dementia. The hospital based data conducted as a thesis work "Prevalence of dementia in old age patients attending Psychiatric clinic in a tertiary care centre over a period of one year" reveals $11.4 \%$ of them had dementia ${ }^{4}$.

Dementia also referred as Neurocognitive disorders (NCDs) as per DSM 5 is a degenerative disease of brain characterized by progressive impairment of memory, communication skills, planning and personal organizational ability, and social skills leading to the progressive decline in the activities of daily living and loss of independence. The cognitive deficits interfere with independence in everyday activities of daily living providing great burden for the family members or care takers ${ }^{5}$.

The diagnosis of dementia is based on the clinical examination, including a mental state examination, and on information from the patient's family, friends and employers. Changes in personality in a person older than 40 years suggest cognitive decline which should be carefully assessed for dementia. There may be denial, or rationalization as the defense mechanism aimed at concealing cognitive deficits. Emotional lability, uninhibited behaviour, silly jokes; or a dull, apathetic, or vacuous facial expression and manner suggest dementia, especially coupled with memory impairment. ${ }^{7}$ The behavioral and psychological symptoms in Dementia(BPSD) is of great concern both for the management point of view and prognosis of the underlying disease process. The Neuropsychiatric symptoms manifestations are often the first noticeable behaviourable changes seen in the patients and the reason for the consultation ${ }^{6}$ BPSD not causes distress for the sufferers but also to the care givers hampering the quality of life and psychological problems. The need of psychological interventions for the better quality of life utmost important. ${ }^{6}$

The manifestation of the symptoms vary depending upon the type of dementia like Alzheimer's type which is the commonest type of dementia followed by vascular dementia, lewy body dementia, Parkinson's disease dementia. Other neurodegenerative conditions like frontotemporal lobar degeneration, traumatic brain injury, HIV infection, Prion disease, Huntington's disease often leads to dementia. The cognitive deficits are also evident in chronic medical conditions so dementia is a heterogonous syndrome which has multiple etiologies ${ }^{7}$.

Risk factors ${ }^{5,7}$

Age is the strongest risk factor for Dementia or NCDs however the risk factors vary depending on the etiological subtype causing dementia. Alzheimer's type dementia is 
relatively seen in later stage of life whereas frontotemporal dementia can be seen throughout the life span. The aged neurons in the brain increase the risk of neurodegenerative and cerebrovascular disease. As per the population monograph of $\mathrm{Nepal}^{8}$ life expectancy at birth for the Nepalese population is 66.6 years against 49.6 in 1981. The overall life expectancy of female is greater than male so the prevalence of dementia specially of Alzheimer type is higher in female genders ${ }^{5}$. This statement holds true even for our context as the life expectancy of female has over taken males in the last 30 years. Life expectancy at birth for female has increased from 48.1 years in 1981 to 67.9 years in $2011^{8}$.

Culture- Related Issues and challenges ${ }^{1,2,5}$

The level of awareness and concerns about the neurocognitive symptoms may vary across the ethinic groups and cultures. There may be differences among the individuals and families about the awareness of cognitive deficits as it can be easily mistaken for age related decline. There is common belief about the cognitive decline and presence of neurocognitive symptoms in advanced age. In most countries, dementia has negative associations for those diagnosed as well as their carers. As per the dementia report:
- $75 \%$ of people with dementia and $64 \%$ of carers identified negative associations about people with dementia in their country.

- $40 \%$ of people with dementia indicated they had been avoided or treated differently because of their diagnosis.

- One in four people with dementia (24\%) admitted to concealing or hiding their diagnosis, citing stigma as the main reason.

- Carers also confront social stigma with one in four (24\%) reporting that they face negative associations and a similar number (28\%) that they have been treated differently or avoided.

- Education, information and awareness were identified as priorities in helping to reduce stigma.

People with dementia are also excluded from everyday life:

In the dementia report released by Alzheimer Disease International $^{1} 40 \%$ of people with dementia reported not being included in everyday life. Nearly $60 \%$ of them indicated that friends are the most likely people to avoid or lose contact after diagnosis followed by family members. One in four people with dementia also indicated they had stopped themselves from forming close relationships after their diagnosis. 
This scenario is almost same in every country, every culture across the globe. So a role of physicians or health care professionals is not only to manage cognitive deficits and BPSD but also to raise awareness about the problem in the community and advocate about the need of National dementia care plan and sensitize the policy makers for long term memory assessment service centers specially in the tertiary referral centers and Zonal hospitals.

\section{Current and future perspectives}

The deterioration of cognitive ability and mental faculties is bothersome for the patients with dementia in early stage of the disease process. When the disease advances and neurodegeneration occurs in most part of the brain patients becomes dependent and may need extensive care. The studies has shown statistically significant benefit with the existing medication like Acetylcholine esterase inhibitors and Memantine when compared with placebo in the global cognitive assessment parameters, reduction of care taker burden and behavioural and Psychological problems ${ }^{9}$. The pre-clinical stage is often a decade long so timely and accurate diagnosis is imperative because progression may be halted or even reversed if appropriate therapy can be provided. Patients often gets benefit from supportive and educational psychotherapy in which the nature and the course of their illness are explained clearly to them and psychodynamic interventions with family members of patients with dementia may be of great assistance in the management of the problem ${ }^{7}$. There are preventive measures available for vascular dementia and other dementias whose etiologies can be predictable but Alzheimer's dementia is still the matter of research so far as its etiology is concerned though the first case was reported by Alois Alzheimer about 109 years back. Studies regarding different types of biomarkers that are suggestive of Alzheimer dementia is underway in the research field which if proven can be helpful in making diagnosis in the preclinical stage ${ }^{10}$.

Status of dementia care and assessment in Nepal

There is least priority from the policy makers for non communicable diseases in low and middle income countries and Nepal isn't an exceptional country. Nepal government has provided some financial concessions in investigations and hospital charges for the geriatric population however they needed to produce citizenship certificate to avail such incentives. The government also provides medication of worth Rs 100,000 if patient meets the criteria for Alzheimer's dementia. This policy in itself is good to start with but it has limited to one type of dementia whereas 
in practice many patients may have mixed dementia or other related problems. There are no nursing homes specially designed for people with dementia or cognitive impairment however there are care homes run by the private sectors. They lack training and expertise to deal patients with dementia who has behavioural problems. The proper dementia assessment is way far behind with respect to the developed country. In B.P Koirala Institute of Health sciences, Dharan we run geriatric mental health and memory clinic once a week and tries our best to provide services and through dementia assessment to the needy population. It is extremely essential for us to have such services or better than this in other parts of the country.

\section{What should be our approach?}

1. Educate the public about dementia

2. Reduce isolation of people with dementia

3. Give people with dementia a voice

4. Involve people with dementia in their local communities

5. Support and educate informal and paid carers

6. Improve the quality of care at home and in care homes

7. Improve dementia training of primary healthcare physicians and other stake holders.

\section{References}

1. http://www.who.int/mental_health/public ations/dementia_report_2012.

2. http://www.who.int/mental_health/public ations/dementia_report_2014.

3. Central Bureau of Statistics, National Population and Housing census 2011 (National report). Government of Nepal, National Planning commissions.

4. Nepal S, Sapkota N, Kumar R, Deo BK; Prevalence of dementia in old age patients attending Psychiatric clinic in a tertiary care centre over a period of one year; thesis done in the department of Psychiatry at B P Koirala Institute of Health Sciences, Dharan, Nepal in Aug 2014.

5. Jeste D. Neurocognitive Disorders. American Psychiatric Association. Diagnostic and Statistical Manual of Mental Disorders: DSM-5. 5th ed. 2013. pp 602-614.

6. Elvish R, Lever S-J, Johnstone $\mathrm{J}$ et al. Psychological interventions for carers of people with dementia: A systematic review of quantitive and qualitative evidence. Counsel Psychother Res.2013;13(2):106-125.

7. Richards SS, Sweet RA. Dementia. In: Sadock BJ, Sadock VA, Ruiz P, eds. Kaplan \& Sadock's Comprehensive Textbook of Psychiatry. 9th edition. 
Philadelphia: Lippincott Williams \& Wilkins; 2009:1167.

8. Central Bureau of Statistics, Population monograph of Nepal, National Planning Commission; 2014 (National report).
9. Singh I, Grossberg GT. Curr Psychiatry 2012; 11 (6): 20-29.

10. Mangialasche et al. Lancet Neurol 2010; 9(\&): 702-716. 